\title{
Evaluation and outcome of patients with chronic non-productive cough using a comprehensive diagnostic protocol
} L P A McGarvey, L G Heaney, J T Lawson, B T Johnston, C M Scally, M Ennis,
D R T Shepherd, J MacMahon combination of investigation and empirical treatment of potential causes based on the anatomical locations of receptors and afferent nerves of the cough reflex have suggested that asthma, post-nasal drip syndrome (PNDS), and gastro-oesophageal reflux (GOR) account for most of the cases..$^{2-6}$ These conditions often co-exist and may simultaneously contribute to cough even when clinically silent. Failure to recognise their contribution may lead to unsuccessful treatment. In this study we investigated patients with chronic non-productive cough (CNPC) for asthma, PNDS, and GOR and then assessed the outcome of treatment based on the diagnoses obtained.

\section{Methods}

SUBJECTS

Patients referred to the chest clinic at Belfast City Hospital with a non-productive cough persisting for more than three weeks as their sole respiratory symptom were recruited to the study. All patients were aged between 18 and 80 years, were lifetime non-smokers, and had a normal chest radiograph and spirometric measurements. Patients with a previous history of chest disease, any systemic disease, an upper respiratory tract infection within the preceding eight weeks, or those taking angiotensin converting enzyme inhibitors were excluded.

The study was approved by the research ethics committee of the Faculty of Medicine, The Queen's University of Belfast and, prior to taking part, written informed consent was obtained from all subjects.

\section{PATIENT EVALUATION}

After history and physical examination, patients were asked to keep a record of morning and evening peak expiratory flow rate (PEFR) for two weeks using a mini-Wright flow meter. A histamine challenge test was performed according to the tidal breathing method of Cockcroft et al. ${ }^{7}$ A computed tomographic (CT) scan of the sinuses was taken in the coronal plane and read by an experienced radiologist (JTL) blinded to the patients' symptoms. Ear, nose and throat (ENT) examination was performed by a consultant ENT surgeon (CMS), also blinded to the patients' symptoms, and graded as follows: 0 , no indication of post-nasal drip; 1, minimal irritation from post-nasal drip; 2, mild irritation from postnasal drip; 3, marked irritation from post-nasal drip; and 4, marked irritation plus mucoid pooling. 
Atopic status was based on history and measurement of total serum IgE and specific serum IgE to four common inhaled allergens (house dust mite, mixed grasses, epithelia mix and cat/dog dander) measured by radioallergosorbent test (RAST; Pharmacia Upjohn).

Ambulatory $\mathrm{pH}$ monitoring of the oesophagus was performed using a Synectics Digitrapper Mark II (Synectics Medical Ltd, Middlesex, UK). After calibration the $\mathrm{pH}$ probe was passed through the nose, advanced, and placed $5 \mathrm{~cm}$ above the upper border of the lower oesophageal sphincter which had been identified by manometry. The patient was then instructed to press the event marker at the onset of a cough and shown how to record the time on a diary card. The oesophageal $\mathrm{pH}$ was recorded for a minimum of 20 hours and the following day information on the digitrapper was downloaded onto an IBM compatible computer. Analysis was performed using the EsopHgram Programme (Gastrosoft Inc, USA). A reflux episode began when the $\mathrm{pH}$ dropped below 4 and ended when the $\mathrm{pH}$ rose above this value. Cough and reflux episodes were deemed to bear a temporal relationship with one another if events occurred simultaneously or within five minutes of one another. ${ }^{8}$

EVALUATION OF COUGH SEVERITY

At the initial consultation a subjective assessment of cough symptom severity was recorded for each patient using a visual analogue scale (VAS). A $100 \mathrm{~mm}$ rating scale was used, one end bearing the statement "no problem whatsoever" and at the other end "unbearable". Patients were then instructed to place a vertical mark on the scale reflecting the severity of their cough. The distance from the "no problem whatsoever" end was measured in $\mathrm{mm}$. The VAS was repeated following treatment. Capsaicin cough challenge was performed as previously described. ${ }^{6}$ Subjects inhaled single breaths of doubling concentrations of capsaicin randomly alternated with breaths of saline. The number of coughs within the minute following the inhalation was counted by the observer. The lowest concentration of capsaicin which elicited at least two coughs (C2) was recorded and the challenge was stopped when the subject coughed five (C5) or more times, or the highest concentration of capsaicin $(500 \mu \mathrm{M})$ had been administered. The challenge was repeated after treatment.

IDENTIFICATION OF DIAGNOSTIC SUBGROUPS Diagnoses were considered on the basis of a consistent history and/or investigation, but were only accepted as contributing to cough when the patient reported satisfactory improvement or complete resolution after a period of diagnosis-specific therapy. A satisfactory improvement was recorded when the patient reported that the cough had subsided to the extent that it was no longer troublesome.

COUGH VARIANT ASTHMA (CVA)

A history of cough at night, during or following exercise, in cold air, or precipitated by aerosols or sprays suggested CVA. A diagnosis of CVA was only accepted when patients were shown to have airway hyperresponsiveness $\left(\mathrm{PC}_{20} \leqslant 8\right.$ $\mathrm{mg} / \mathrm{ml} \mathrm{histamine})^{7}$ and reported cough resolution or satisfactory improvement following specific treatment which was as follows: inhaled beclomethasone dipropionate $1 \mathrm{mg}$ twice daily (via a Volumatic spacer) together with inhaled salbutamol, two puffs as required for at least eight weeks. At review patients still symptomatic were given a two week course of oral steroids (40 mg daily for seven days then $30 \mathrm{mg}$ daily for seven days). Inhaled steroids were then recommenced until further review.

POST-NASAL DRIP SYNDROME (PNDS)

A diagnosis of post-nasal drip syndrome (PNDS) was considered when patients described the sensation of "having something drip down into their throat" and/or the need to frequently clear their throat. This was further divided into rhinitis (history of one or more of nasal congestion, rhinorrhoea, sneezing, and itching $^{9}$ ), sinusitis (history of purulent nasal discharge, headache or facial pain), and rhinosinusitis when the history suggested both conditions.

A positive ENT examination (grade $\geqslant 1$ ) suggested the potential contribution of PNDS to the cough. A coronal CT scan of the sinuses showing mucosal thickening, air fluid level, or sinus opacification was consistent with sinusitis. ${ }^{10}$ Treatment for PNDS was given as follows: for rhinitis a topical decongestant (xylometazoline hydrochloride $0.1 \%$ ) two drops per nostril twice daily was given for one week plus intranasal fluticasone propionate $100 \mu \mathrm{g}$ (two sprays) per nostril twice daily for at least eight weeks), and for sinusitis oral cefaclor $500 \mathrm{mg}$ twice daily was given for two weeks plus intranasal fluticasone propionate $100 \mu \mathrm{g}$ (two sprays) per nostril twice daily for at least eight weeks. Patients with history suggestive of nasal allergy and who were atopic were treated with oral terfenadine $120 \mathrm{mg}$ at night.

GASTRO-OESOPHAGEAL REFLUx (GOR)

Cough associated with dyspepsia, occurring after meals, when stooping, or when supine was suggestive of reflux associated cough. Patients were identified as having abnormal reflux on 24 hour oesophageal $\mathrm{pH}$ monitoring if they exceeded the 95th percentile for percentage total time with $\mathrm{pH}<4$ of $>4.8 \%$, percentage upright time with $\mathrm{pH}<4$ of $>6.2 \%$, and percentage supine time with $\mathrm{pH}<4$ of $>1.2 \%$ for 20 normal volunteers. ${ }^{11}$ Treatment consisted of dietary advice (weight loss, low fat/low calorie foods, avoidance of late night snacks), postural advice (avoidance of stooping, elevation of head of bed), and oral omeprazole $20 \mathrm{mg}$ at night for eight weeks. If symptoms were persistent omeprazole was increased to $20 \mathrm{mg}$ twice daily until review.

MULTIPLE CAUSES

A patient was identified as having multiple causes for cough when the history and/or investigation suggested more than one cause and cough only responded to combination therapy. 
Table 1 Characteristics of diagnostic subgroups

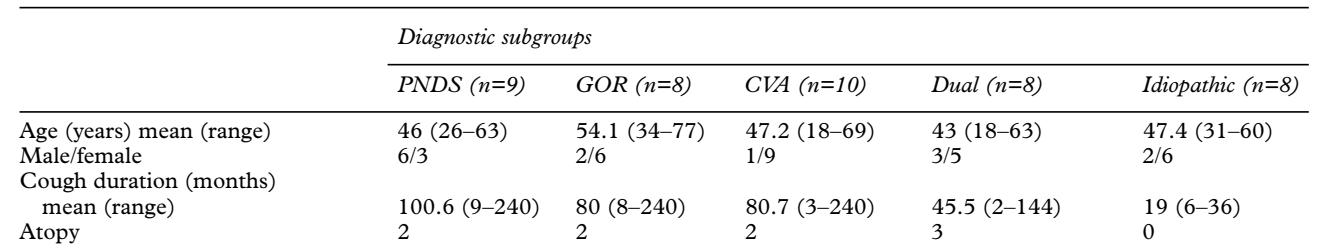

PNDS = post-nasal drip syndrome; GOR = gastro-oesophageal reflux; CVA = cough variant asthma.

Atopic status based on history and total serum IgE (normal $<120 \mathrm{kU} / \mathrm{l}$ ) and specific serum IgE to four common inhaled allergens (house dust mite, mixed grasses, epithelia mix and cat/dog dander) (score $<1$ rated negative) measured by radioallergosorbent test (RAST).

IDIOPATHIC

Patients were identified as idiopathic if the cough persisted despite sustained treatment (at least four months) directed against the presumed cause or causes, or if they had an unremarkable history and normal investigations and the cough resolved spontaneously or persisted despite empirical treatment for CVA, PNDS, and GOR.

\section{STATISTICS}

The components of the diagnostic protocol were described using probability statistics in terms of positive predictive value. ${ }^{12} \mathrm{~A}$ test result was deemed a true positive or false positive based on the outcome of specific treatment. The probability that the disease was present when the test was positive was defined as the positive predictive value. Log C2 2 and $\log$ C5 values were compared before and after treatment using the Wilcoxon signed rank test.

\section{Results}

PATIENT CHARACTERISTICS

Forty three patients (29 women) were evaluated using the protocol. The mean age was 47.5 years (range 18-77) and the mean duration of cough for all patients was 67 months (range 2-240). The mean time from initial interview to starting treatment was 63.9 days (range 28-140). When compared with all the coughers where a cause was identified, the idiopathic coughers had significantly shorter duration of symptoms prior to referral (mean (SD) 19.0 (10.3) months versus 77.6 (81.3) months, $\mathrm{p}<0.05)$.

\section{DIAGNOSTIC SUBGROUPS}

On the basis of successful response to treatment, a cause for the cough was identified in 35 patients $(82 \%)$. A single cause for cough was found in 27 individuals and in eight patients the cough was due to two causes. No patient with three or more causes was identified. The
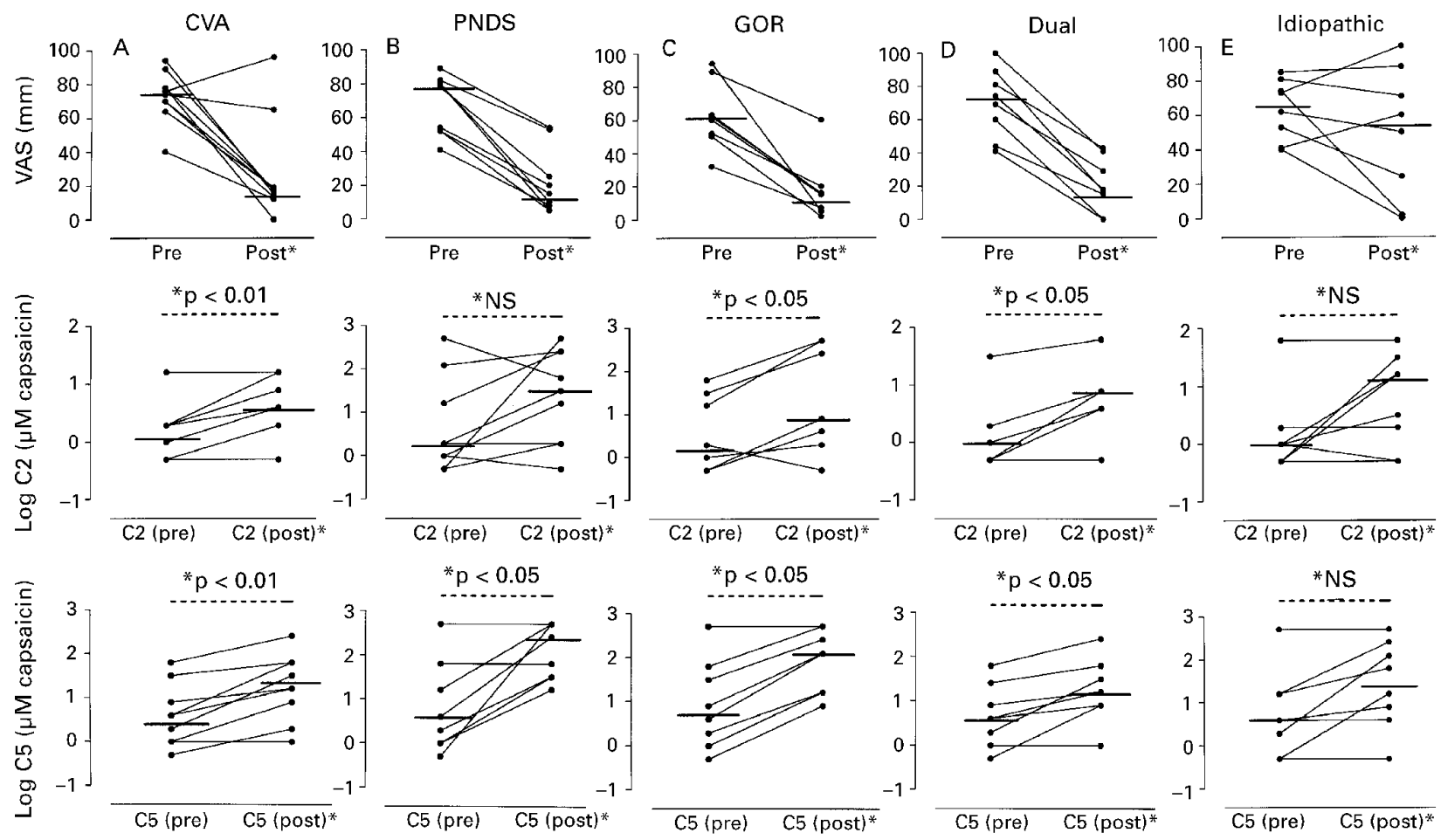

Figure 1 Visual analogue scale (VAS) log capsaicin concentration to elicit two coughs (C2) and five coughs (C5) in (A) 10 patients with cough variant asthma (CVA), (B) nine patients with post-nasal drip syndrome (PNDS), (C) eight patients with gastro-oesophageal reflux (GOR), (D) eight patients with dual aetiology, and (E) eight patients with idiopathic cough before and after treatment. Horizontal lines represent median values. 
Table 2 Predictive value of symptom characteristics obtained from history

\begin{tabular}{llll}
\hline & $\begin{array}{l}\text { No. with } \\
\text { positive history }\end{array}$ & $\begin{array}{l}\text { No. correctly } \\
\text { identified }\end{array}$ & $\begin{array}{l}\text { Positive } \\
\text { predictive value (\%) }\end{array}$ \\
\hline $\begin{array}{l}\text { Asthma (nocturnal cough, precipitated by } \\
\text { cold air, exercise, aerosols) }\end{array}$ & 27 & 15 & 56 \\
$\begin{array}{l}\text { PNDS (throat clearing, sensation of post } \\
\text { nasal drip, nasal discharge, previous }\end{array}$ & & & \\
$\quad$ sinusitis) & 27 & 14 & 52 \\
GOR (dyspepsia, cough worse after meals) & 20 & 8 & 40
\end{tabular}

PNDS = post-nasal drip syndrome; GOR = gastro-oesophageal reflux.

range, frequency, and characteristics of the subgroups identified are displayed in table 1.

VISUAL ANALOGUE SCORES

The visual analogue scores after treatment were lower than pretreatment values for each diagnostic subgroup, except for the idiopathic cases where the values did not differ significantly before and after treatment (fig 1A-E).

CAPSAICIN COUGH CHALLENGE

In all treatment success subgroups $\log \mathrm{C} 2$ and $\log \mathrm{C} 5$ values after treatment were significantly higher than pretreatment values except in the PNDS group where $\log$ C2 2 was not significantly different (fig 1A-D). There was no significant difference in the idiopathic group, although in four patients the capsaicin cough threshold increased (fig 1E).

\section{CHARACTERISTICS OF DIAGNOSTIC PROTOCOL}

History

Table 2 shows the predictive values for the history in relation to response to treatment. All patients with CVA reported one or more characteristic features of asthmatic cough, although the symptom was not unique to CVA (15 of 27 $(56 \%)$ with positive symptoms). A history of symptoms of PNDS identified a true positive in only half the cases (positive predicted value $=$ $52 \%)$. Clinically silent GOR was present in $46 \%$ of patients with cough associated with reflux while a suggestive history, when present, had a positive predictive value of only $40 \%$.

\section{Examination/investigation}

The positive predictive values for the components of the diagnostic protocol are displayed in table 3. On the basis of a positive investigation directing successful treatment, histamine challenge was the most useful with a positive predictive value of $88 \%$. A positive predictive value of $68 \%$ for 24 hour oesophageal $\mathrm{pH}$ monitoring was obtained and sinus CT imaging and ENT examination had values of $67 \%$ and $63 \%$, respectively.

Histamine challenge testing and 24 hour oesophageal $\mathrm{pH}$ monitoring, when negative, effectively excluded CVA and GOR, respec-

Table 3 Positive predictive value of specific components of diagnostic protocol (using successful response to therapy as the outcome)

\begin{tabular}{llll}
\hline Test & $\begin{array}{l}\text { No. of positive } \\
\text { tests }\end{array}$ & $\begin{array}{l}\text { No. responding to } \\
\text { relevant treatment }\end{array}$ & $\begin{array}{l}\text { Positive predictive } \\
\text { value (\%) }\end{array}$ \\
\hline Histamine challenge & 17 & 15 & 88 \\
24 h oesophageal pH monitoring & 19 & 13 & 68 \\
ENT examination & 16 & 10 & 63 \\
CT scan sinuses & 18 & 12 & 67
\end{tabular}

ENT $=$ ear, nose and throat; CT = computed tomography. tively, in all cases. In $88 \%$ of cases where sinus CT scanning was negative, a cause other than PNDS was identified for the cough. A normal ENT examination reliably ruled out PNDS as a possible cause for the cough in $77 \%$ of cases.

The data on peak expiratory flow monitoring could not be processed as 12 patients either failed to return or to complete their diary.

\section{Outcome}

Of the 15 patients identified with CVA, six later developed wheeze and breathlessness despite sustained treatment. PNDS, either alone or in combination, was diagnosed in 15 patients. Sinusitis or rhinosinusitis appeared to contribute to the cough in the majority of patients (14 cases) rather than rhinitis in isolation (one case). Relapse of symptoms on stopping treatment was frequently seen in the PNDS group (six patients).

One patient with GOR failed to tolerate omeprazole due to diarrhoea, despite control of the cough. He remained symptom free on an $\mathrm{H}_{2}$ antagonist. A further patient required 20 $\mathrm{mg}$ twice daily of omeprazole for symptom relief and relapsed on stopping treatment. Among the four cases of idiopathic cough, in whom the capsaicin threshold increased, the cough resolved spontaneously in three individuals. The other, however, had a persistent cough despite sustained courses of empirical treatment.

\section{Discussion}

In this selected group of patients with chronic cough, asthma, PNDS, and GOR - either alone or in combination-contributed to the cough in $80 \%$ of cases. Resolution of symptoms following diagnosis-specific treatment was associated with a significant increase in the capsaicin threshold.

Treatment of CVA, either alone or in combination, led to resolution of symptoms in 15 patients $(35 \%)$ and, although they all described cough precipitated by cold air, exercise and/or aerosols, this feature in the history was not uncommon among non-asthmatic patients. The degree of bronchial hyperreactivity in subjects with CVA was mild (geometric mean $\mathrm{PC}_{20}$ $=3.44$ (range $0.04-8 \mathrm{mg} / \mathrm{ml})$ ). Histamine challenge testing had a positive predictive value of $88 \%$ which suggests that not all patients with cough and bronchial hyperresponsiveness have CVA. We therefore reinforce the conclusions of a recent study which suggested that asthma could only be diagnosed when positive results on bronchial challenge were followed by a favourable response of the cough to asthma therapy. ${ }^{13}$ A negative histamine challenge ruled out asthma as a cause for cough and obviated the need for a trial of inhaled steroids and bronchodilators, highlighting its utility in any diagnostic protocol for cough. Despite sustained therapy, six patients later developed new symptoms of dyspnoea and wheeze more typical of "classical" asthma, requiring courses of oral steroids in addition to their maintenance inhaled therapy. This is consistent with a reported follow up of 16 patients with CVA 
over 3-5 years where $50 \%$ required continued treatment and the majority developed wheeze or breathlessness. ${ }^{14}$

PNDS is the most frequent cause for chronic cough and the use of empirical antihistamine/ decongestant therapy in all patients with chronic cough has been advocated. ${ }^{5}$ Our observation that PNDS, either alone or in combination, contributed to cough in $35 \%$ of the patients concurs with this. We recognise, however, the limitations of relying on a characteristic history to help direct treatment. Although in one study "silent" post-nasal drip was reported in $20 \%$ of cases, ${ }^{5}$ all our patients with PNDS had the characteristic symptoms; an additional 12 patients reported post-nasal drip but their cough responded to treatment directed at an alternative cause. ENT examination of our patients accurately predicted outcome to specific treatment in $63 \%$ of cases and, when normal, PNDS was ruled out in over $75 \%$ of cases. While the "cobblestone" appearance of the mucosa of the nasopharynges and oropharynges has been described, we observed this pattern in only one patient and suggest it is a very uncommon finding in the routine examination of patients with chronic cough. The positive predictive value of CT scanning of the sinuses was $67 \%$ and, when negative, it ruled out PNDS as a possible cause in almost $90 \%$ of cases. However, when the ENT examination and sinus CT scan are performed and interpreted together, no additional benefit is achieved in accuracy of diagnosis. We would suggest that a full ENT examination should be performed in all patients with CNPC and CT sinus scanning reserved for those patients with a suggestive history but normal ENT inspection or persistent cough despite sustained treatment. Although PNDS clearly contributes to cough in many cases, in some it merely co-exists and plays little part in the symptom. This is consistent with a previous observation that eight patients with rhinitis which cleared with treatment were still troubled with a cough. ${ }^{6}$

There is little dispute that gastro-oesophageal reflux is a causative factor in chronic cough. However, the absence of typical symptoms of dyspepsia may result in the dismissal of GOR as the cause of the cough. In $46 \%$ of our patients with GOR associated cough, typical acid reflux symptoms were absent and abnormal acid reflux on 24 hour oesophageal $\mathrm{pH}$ monitoring was the only positive finding. The exact mechanism by which acid reflux causes cough is unclear, although recording the temporal correlation of cough episodes with reflux events may help to elucidate this. In our hands a negative 24 hour $\mathrm{pH}$ study ruled out GOR as a possible cause of cough. This is in agreement with two studies, one prospective ${ }^{8}$ and the other retrospective, ${ }^{15}$ which reported negative predictive values of $100 \%$ and $90 \%$, respectively. Interpretation of a positive result is more difficult and our finding of a positive predictive value of only $68 \%$ highlights the fact that, among patients with cough, there is a significant amount of co-existent GOR which is not directly related to the cough. At present, 24 hour $\mathrm{pH}$ monitoring remains the best method for detecting acid reflux ${ }^{16}$; however, its place in the routine evaluation of patients with chronic cough requires further study.

Despite this comprehensive protocol, we were unable to identify a cause for cough in eight patients $(18 \%)$. In three patients with no clear diagnosis after history and examination, and in the setting of negative examination, the cough disappeared spontaneously. In the remaining five the cough persisted despite multiple courses of treatment directed at all possible causes. It is possible, given the significantly shorter duration of cough prior to referral observed in the idiopathic subgroup, that cough had persisted following a subclinical viral upper respiratory tract infection, particularly in the three cases where the cough resolved spontaneously. Although most coughs resolve in 2-3 weeks following an upper respiratory tract infection, ${ }^{17}$ some may persist for many months and years thereafter.

In this study we have used the capsaicin cough challenge to provide some objective support for the change, if any, in the cough severity. As a research tool the challenge is safe, easy to perform, and has been reported to have good reproducibility. ${ }^{18}$ In clinical practice, because the enhanced response to capsaicin is non-specific with respect to the aetiology of cough, its routine use within a diagnostic protocol is limited. However, the reduction in tussive response to capsaicin on resolution of cough suggests that it could be used to assess the efficacy of treatment.

This study describes the outcome of a comprehensive protocol applied to all patients referred with chronic cough. Although conducted in a selected series of patients, all nonsmokers with normal chest radiographs and spirometric measurements, our findings support the currently held view that identifying a cause and then directing treatment at that cause is the best approach. We would suggest that the evaluation of such patients should involve careful history taking and examination, with particular attention to the upper airway. We consider that the routine addition of sinus CT scanning is not indicated but should be reserved for refractory cases which may require surgical referral. Before prescribing treatment bronchial provocation testing and 24 hour oesophageal $\mathrm{pH}$ monitoring should be considered in all cases as a negative investigation rules out disease and may prevent unsuccessful courses of empirical treatment. In addition, 24 hour oesophageal $\mathrm{pH}$ monitoring remains the best means of identifying silent but clinically significant acid reflux. Furthermore, this protocol aids the accurate direction of treatment and may reduce the number of review appointments after failed empirical treatment. However, while asthma, PNDS and GOR remain the most important causes to consider, a group with persistent symptoms and no identifiable aetiology remains.

We acknowledge support from the Northern Ireland Chest Heart and Stoke Association. We would like to thank the staff in the Chest Clinic, Mrs J Megarry in the Pulmonary Function Laboratory at the Belfast City Hospital, and Sister E Crawford Laboratory at the Belfast City Hospital, and Sister E Crawford
in the Department of Surgery for their co-operation and assistin the Department of Surgery for their co-operation and
ance. Mrs I Murray is thanked for her secretarial help. 
1 McGarvey LPA, Heaney LG, MacMahon J. A retrospective survey of diagnosis and management of patients presenting
with chronic cough to a general chest clinic. Int f Clin Pract with chron

2 Irwin RS, Corrao WM, Pratter MR. Chronic persistent cough in the adult: the spectrum and frequency of cases and successful outcome of specific therapy. Am Rev Respir Dis 1981;123:414-7.

3 Irwin RS, Curley FJ, French CL. Chronic cough: the spectrum and frequency of causes, key components of the diagnostic evaluation and outcome of specific therapy. Am Rev Resp Dis 1990;141:640-7.

4 Poe HR, Harder RV, Israel RH, et al. Chronic persistent cough: experience in diagnosis and outcome using an anatomic diagnostic protocol. Chest 1989;95:723-7.

5 Pratter MR, Bartter T, Akers S, et al. An algorithmic approach to chronic cough. Ann Intern Med 1993;119:977-83.

6 O'Connell F, Thomas VE, Fuller RW, et al. Cough sensitivity to inhaled capsaicindecreases with successful treatment of chronic

7 Cockcroft DW, Killin DN, Malon JA, et al. Bronchial reactivity to inhaled histamine: a method and clinical survey. Clin Allergy 1977;7:235-40

8 Ing AJ, Ngu MC, Breslin ABX. Chronic persistent cough and gastro-oesophageal reflux. Thorax 1991;46:479-83.

9 Lund VJ, Aaronson DW, Bousquet J, et al. Internationa consensus report on the diagnosis and management of rhinitis. Allergy 1994;49(Suppl 19):5-34.
10 Shapiro GG, Rachelefsky GS. Introduction and definition of sinusitis. F Allergy Clin Immunol 1992;3:417-8.

11 Johnston BT, McFarland RJ, Collins JSA, et al. The symptom index. A useful marker of gastro-oesophageal reflux disease. Br $\mathcal{F}$ Surg 1992;79:1054-5.

12 Grimer PF, Mayewski RJ, Mushlin AI, et al. Selection and interpretation of diagnostic tests and procedures: principles and applications. Ann Intern Med 1981;94:553-60.

13 Irwin RS, French CT, Smyrnios NA, et al. Interpretation of positive results of a methacholine inhalation challenge and 1 week of inhaled bronchodilator use in diagnosing and treating cough variant asthma. Arch Intern Med 1997;157: 1981-7.

14 Braman S, Pordy W, Corrao W, et al. Cough variant asthma: a 3-5 year follow up (abstract). Am Rev Respir Dis 1982;125:133.

15 Vaezi MF, Richter JE. Twenty four hour ambulatory eosophageal $\mathrm{pH}$ monitoring in the diagnosis of acid related cough. Southern Med F 1997;90:305-11.

16 Mattox HE, Richter JE. Prolonged ambulatory esophageal $\mathrm{pH}$ monitoring in the evaluation of gastroesophageal reflux disease. Am f Med 1990; 89:345-56.

17 Curley FJ, Irwin RS, Pratter MR. Cough and the common cold. Am Rev Respir Dis 1988;138:305-11.

18 O'Connell F, Thomas VE, Fuller RW, et al. Reproducibility of capsaicin-induced cough in humans (abstract). Eur Respir F 1995;8:96s. 